\title{
Mechanisms underlying the promotion of osteosarcoma cell proliferation and invasion by IncRNA PBB12
}

\author{
TIANWEN MA ${ }^{1 *}$, AIPING LIU ${ }^{2 *}$, DONGWEN XU ${ }^{2}$ and TAO ZHANG ${ }^{2}$ \\ Departments of ${ }^{1}$ Orthopedics and ${ }^{2}$ Laboratory Medicine, Huashan Hospital, Fudan University, Shanghai 200040, P.R. China
}

Received August 12, 2019; Accepted November 29, 2019

DOI: $10.3892 /$ or.2019.7451

\begin{abstract}
PBB12, a long noncoding RNA (lncRNA), has not been reported to be involved in the progression of osteosarcoma to date. According to our research data, it was found that osteosarcomapatients withahighPBB12 levelfrequently showed increased metastasis. Mechanistic research demonstrated that PBB12 can competitively bind to hsa-miR-204-5p by acting as a microRNA sponge. Furthermore, PBB12 intervenes in the Kruppel-like factor 4 (KLF4)/hsa-miR-204-5p/activating transcription factor 2 (ATF2) pathway and affects the proliferation and invasion of osteosarcoma cells. High PBB12 expression was founld to lead to the loss of function of the cancer suppressors KLF4 and hsa-miR-204-5p; these effects are the intrinsic reason why osteosarcoma patients with high levels of PBB12 often develop tumor metastases. In vitro functional experimental data showed that in the osteosarcoma cell lines MG63 and SAOS-2, PBB12 silencing and overexpression significantly increases or reversed the inhibitory effect of KLF4 on the proliferation and invasion of tumor cells, respectively. The involvement of the interaction between PBB12 and the KLF4/hsa-miR-204-5p/ATF2 pathway in osteosarcoma progression was reported for the first time, and these data provide important theoretical support for gene therapy targeting KLF4 and hsa-miR-204-5p. The great significance of this study is that a high or low genetic background level of PBB12 in osteosarcoma patients may serve as an important marker for screening patients for a high risk of tumor metastasis. Furthermore, for osteosarcoma patients with high PBB12 expression, the primary task for all accurate personalized clinical treatment programs may be the determination of how to effectively knock down PBB12 in tumor bodies.
\end{abstract}

Correspondence to: Dr Tao Zhang, Department of Laboratory Medicine, Huashan Hospital, Fudan University, 12 Central Urumqi Road, Shanghai 200040, P.R. China

E-mail: 3165735860@qq.com

${ }^{*}$ Contributed equally

Key words: PBB12, hsa-miR-204-5p, KLF4, ATF2, osteosarcoma

\section{Introduction}

Osteosarcoma (OS), a common malignant tumor originating from bone tissue, commonly presents in children and adolescents. Approximately one-third of OS patients survive less than 5 years, and the metastasis of osteosarcoma is the main factor contributing to this poor prognosis. Therefore, the development of innovative therapies for OS is an urgently needed task in the field of OS research $(1,2)$. In recent years, basic studies have shown that long noncoding RNAs (lncRNAs) can play a regulatory role in tumor progression by extensively regulating proliferation, invasion and metastasis in tumor cells (3). IncRNAs, as competing endogenous RNAs, interact with microRNAs (miRNAs) to regulate target genes to play important roles in the onset and development of cancers $(4,5)$. Along with miRNAs, lncRNAs have become a 'hot' topic in tumor research due to their antitumor or tumorigenic effects and their potentially important role in tumor diagnosis, treatment and prognosis determination.

Although previous studies have shown that abnormal lncRNA expression occurs in the progression of OS, we still require more in-depth research in regards to the mechanisms of OS progression (6-8). In the present study, we discovered and are reporting for the first time lncRNA PBB12 (named by us), which is closely related to the proliferation and invasion of OS cells. Our study not only showed that PBB12 knockdown can effectively inhibit the proliferation, invasion and metastasis of OS cells by restoring the function of the tumor-suppressor gene Kruppel-like factor 4 (KLF4) but also showed that PBB12 is likely to become a clinical screening marker for patients at high risk for OS metastasis.

\section{Materials and methods}

Cell culture. The OS cell lines MG63 (TCHu124) and SAOS-2 (TCHu114) were obtained from the Cell Bank of the Chinese Academy of Sciences (CBCAS; Shanghai, China) and maintained in minimal essential medium (MEM; cat.no. 11095-072; Thermo Fisher Scientific, Inc.) supplemented with $10 \%$ fetal calf serum (FBS; cat. no. 10099-141; Thermo Fisher Scientific, Inc.). 293TN cells (a lentivirus-producing cell line) were purchased from System Biosciences (cat. no. LV900A-1) and maintained in Dulbecco's modified Eagle medium (DMEM; cat. no. 10569-044; Thermo Fisher Scientific, Inc.) supplemented with $10 \%$ FBS and were passaged via $0.25 \%$ trypsin 
digestion (cat. no 25200056; Thermo Fisher Scientific, Inc.). All cells were adherently grown in $5 \% \mathrm{CO}_{2}$ at $37^{\circ} \mathrm{C}$ and in saturated humidity.

Tumor tissues. OS tumors with or without metastasis and the adjacent normal tissues (within a $2.5-\mathrm{cm}$ radius from the edge of the tumor) were collected from 24 patients at the Department of Orthopedics, Huashan Hospital between October 2017 and July 2018 (detailed patient information is presented in Table I). The selected patients were required to be under 25 years of age; both male and female patients were diagnosed for the first time, and no patient was treated by chemotherapy or radiotherapy prior to surgery. This study was approved by the Ethics Review Committee of Huashan Hospital Affiliated to Fudan University (2016-037), and written informed consent was obtained from all participants and/or their guardians. The tissues were used for total RNA extraction and real-time PCR for the measurement of PBB12, hsa-miR-204-5p and activating transcription factor 2 (ATF2) mRNA levels. Total protein extraction and western blotting were used to assess Kruppel-like factor (KLF4) and ATF2 expression.

Recombinant lentivirus preparation. An siRNA (5'-GCTCGG TGTAAAGGGAGGG-3') specific for PBB12 was chosen, and the shRNA oligonucleotide DNA was synthesized and cloned into the shRNA vector pSIH1-H1 (SI501A-1, System Biosciences) after double-strand annealing. A nonsense sequence of siRNA (5'-GGTAGGTGGGCAGCTGAGA-3') was used as a negative control (NC). The constructed vectors were named pSIH1-shRNA-PBB12 and pSIH1-NC. The coding sequence (CDS) of the KLF4 gene $(1,542 \mathrm{bp})$ was amplified using the primers 5'-GGAATTCGCCACCATGAG GCAGCCACCTGGCG-3' and 5'-CGGGATCCTTAAAAA TGCCTCTTCATGTGTAAGG-3' using human complementary DNA (cDNA) as the template. The PCR product was digested with EcoRI and BamHI and cloned into the expression vector pcDH-GFP (CD511B-1, System Biosciences) to construct the recombinant vector pcDH-KLF4. PcDH-PBB12 was constructed by the same method. The pair of PCR primers were 5'-GGAATTCTAATAGAATGATTTTTATT-3' (forward) and 5'-CGGGATCCGAGGAAGGAGGGCGCA GGGCA-3' (reverse), and these primers produced a 1,121-bp PCR product. All recombinant vectors were sequenced, and plasmid DNA was prepared using an EndoFree Plasmid Kit (cat. no. 12362; Qiagen).

A total of $1 \times 10^{6} 293 \mathrm{TN}$ cells each were seeded into $10-\mathrm{cm}$ dishes in $10 \mathrm{ml}$ DMEM containing 10\% FBS and were cultured overnight under normal conditions. Two micrograms of each recombinant vector (pcDH-KLF4, pshRNA-PBB12, pshRNA-NC or pcDH-PBB12) and $10 \mu \mathrm{g}$ of the pPACK Packaging Plasmid Mix (LV500A-1; System Biosciences) were cotransfected using the cationic liposome transfection reagent Lipofectamine 2000 (cat. no. 11801169; Invitrogen). The medium was fully replaced with DMEM plus $1 \%$ FBS before transfection. After $48 \mathrm{~h}$, the supernatant was harvested and cleared by centrifugation at $5,000 \mathrm{xg}$ at $4^{\circ} \mathrm{C}$ for $10 \mathrm{~min}$ and then passed through a $0.45-\mu \mathrm{m}$ PVDF membrane (cat. no. IPVH00010, Millipore). The titer of the virus was determined by a gradient dilution method. The recombinant lentiviruses were named Lv-KLF4, Lv-shRNA-PBB12, Lv-NC and Lv-PBB12.

\section{Luciferase assay}

Verification of the seed region of hsa-miR-204-5p in ATF2 3'UTR. Part of the human ATF2 (NM_001880.4) 3'UTR (196 bp) containing the theoretical seed region was amplified from human cDNA with the primers 5'-GCTCTAGAA ACCTGCAGTACAACAGTT-3' (forward) and 5'-GCTCTA GAA ATCAGTCTTTTTCCAGAGAC-3' (reverse). The PCR product was digested with $X b a \mathrm{I}$ and cloned into the pGL3-promoter vector (E1761, Promega) at the downstream position of the luciferase gene to construct pGL3-wt-ATF2, which carried the wild-type seed region of hsa-miR-204-5p; the seed region was mistranslated from 5'-AAGGGA A-3' to 5'-GAGAAAG-3' to construct pGL3-mt-ATF2 and carried the mutant hsa-miR-204-5p seed region. The hsa-miR-204-5p-mimic (5'-UUCCCUUUGUCAUCCUAU GCCUTT-3'), hsa-miR-204-5p-inhibitor (5'-AGGCAUAGG AUGACA AAGGGA ATT-3') and hsa-miR-204-5p-NC (5'-UGUAUUCUCUCUCACUUCCCUGTT-3') were obtained from Shanghai GenePharma (Shanghai, China). 293TN cells were cotransfected with the miR-204-mimic, inhibitor, or NC and pGL-wt-ATF2 or pGL3-mt-ATF2 using Lipofectamine 2000 according to the manufacturer's instructions. Forty-eight hours after transfection, the cells were harvested, and luciferase assays were performed using a Dual-Luciferase Reporter Assay System (cat. no. E1910; Promega).

The experiment to observe the effect of PBB12 depletion on the inhibition of luciferase by the hsa-miR-204-5p mimic was carried out in $293 \mathrm{TN}$ cells by using the same method used for the seed region validation. We overexpressed PBB12 by transfecting pcDH-PBB12 in 293TN cells cotransfected with miR-204-5p mimics and pGL3-wt-ATF2. If overexpression of PBB12PBB12 was able to upregulate intracellular luciferase activity, it would indicate that PBB12 can significantly attenuate the negative regulation of miR-204-5p on ATF2.

Validation of the transcription factor binding site (TFBS) of KLF4 in the promoter of pri-miR-204-5p. We first searched for the location of the precursor of hsa-miR-204-5p (pri-miR-204) in the human genome and selected a $2.5 \mathrm{~kb}$ DNA sequence upstream of the transcription start site as the promoter region. Then, we predicted the promoter sequence using Promoter 2.0 software (DTU Health Tech). The KLF4 TFBS in the pri-miR-204 promoter was predicted by 'JASPAR' (9). The promoter of pri-miR-204 (312 bp) was amplified using human genomic DNA as the template with the primers 5'-GGGGTA CCCTAGCACTCACTCAGTGACT-3' (forward) and 5'-CCC AAGCTTGGGAATAGTTCTGGGTCCAAACCATGTGAC-3' (reverse). The PCR product was digested with $K p n I$ and HindIII and was cloned into the luciferase reporter vector pGL3-Enhancer (E1771; Promega) at a position upstream of the luciferase gene to construct pGL3-TFBS(wt)-miR-204, which carried the wild-type TFBS. Then, the TFBS in the pGL3-TFBS(wt)-miR-204 vector was mutated from 5-CACC-3' to 5'-CCAC-3' to construct pGL3-TFBS(mt)-miR-204, which carried a mutated TFBS. The experiment was carried out in $293 \mathrm{TN}$ cells, and the detection and analysis methods used for transfection and the measurement of luciferase activity assay were the same as those used above. 
Table I. Clinicopathological features of the 24 patients with or without metastatic osteosarcoma.

\begin{tabular}{|c|c|c|c|c|}
\hline $\begin{array}{l}\text { Patient } \\
\text { no. }\end{array}$ & Sex & $\begin{array}{c}\text { Age } \\
\text { (years) }\end{array}$ & $\begin{array}{l}\text { TNM } \\
\text { stage }\end{array}$ & $\begin{array}{l}\text { Site of } \\
\text { metastasis }\end{array}$ \\
\hline 1 & $\mathbf{M}$ & 12 & T2NxM0 & None \\
\hline 2 & $\mathbf{F}$ & 20 & T2N1M0 & None \\
\hline 3 & $\mathbf{F}$ & 16 & T1NxM0 & None \\
\hline 4 & $\mathbf{M}$ & 12 & T2N1M0 & None \\
\hline 5 & $\mathbf{F}$ & 11 & T1N0M0 & None \\
\hline 6 & $\mathbf{F}$ & 18 & T1N1M0 & None \\
\hline 7 & $\mathbf{M}$ & 15 & T2NxM0 & None \\
\hline 8 & $\mathbf{M}$ & 16 & T1N0M0 & None \\
\hline 9 & $\mathbf{F}$ & 15 & T2NxM0 & None \\
\hline 10 & $\mathbf{M}$ & 13 & T1N1M0 & None \\
\hline 11 & $\mathbf{F}$ & 16 & T2NxM0 & None \\
\hline 12 & $\mathrm{~F}$ & 18 & T2N0M0 & None \\
\hline 13 & $\mathrm{~F}$ & 14 & $\mathrm{~T} 2 \mathrm{~N} 2 \mathrm{M} 1$ & Liver \\
\hline 14 & $\mathrm{M}$ & 16 & $\mathrm{~T} 2 \mathrm{~N} 1 \mathrm{M} 1$ & Lung \\
\hline 15 & $\mathrm{~F}$ & 12 & $\mathrm{~T} 2 \mathrm{NxM} 1$ & Bone \\
\hline 16 & $\mathrm{M}$ & 15 & $\mathrm{~T} 2 \mathrm{~N} 2 \mathrm{M} 1$ & Bone \\
\hline 17 & M & 17 & $\mathrm{~T} 2 \mathrm{~N} 1 \mathrm{M} 1$ & Kidney \\
\hline 18 & $\mathrm{~F}$ & 11 & T3N1M1 & Lung \\
\hline 19 & M & 13 & $\mathrm{~T} 2 \mathrm{~N} 1 \mathrm{M} 1$ & Brain \\
\hline 20 & $\mathrm{M}$ & 14 & T1NxM1 & Brain \\
\hline 21 & $\mathrm{~F}$ & 21 & T3N1M1 & Lung \\
\hline 22 & $\mathrm{~F}$ & 15 & T3N2M1 & Kidney \\
\hline 23 & $\mathrm{~F}$ & 12 & $\mathrm{~T} 2 \mathrm{~N} 1 \mathrm{M} 1$ & Lymph node \\
\hline 24 & $\mathrm{M}$ & 14 & $\mathrm{~T} 2 \mathrm{~N} 1 \mathrm{M} 1$ & Liver \\
\hline
\end{tabular}

A total of 24 pairs of osteosarcoma tumors and adjacent normal tissues were obtained from patients from the Orthopedics Department of Huashan Hospital between October 2017 and July 2018. Clinical stage categories were determined based on the 7th edition of the TNM guidelines. F, female; M, male. Patients presenting with no metastasis are in bold print.

Cell function experiment. MG63 and SAOS-2 OS cells were trypsinized and seeded into 96 -well plates at a density of $1 \times 10^{5}$ cells per well $72 \mathrm{~h}$ after being infected with the recombinant lentiviruses (Lv-NC, Lv-KLF4, Lv-PBB12+Lv-KLF4 or Lv-shRNA-PBB12+Lv-KLF4). The cells were cultured under normal conditions, and cell viability was detected using a Cell Counting Kit-8 assay (CCK-8, CK04; Dojindo Molecular Technologies, Inc.) at 24, 48, and $72 \mathrm{~h}$. Cell invasion experiments were performed using the QCM ECMatrix Cell Invasion Assay, 24-well $(8 \mu \mathrm{m})$, fluorimetric (ECM554; Chemicon International) according to the manufacturer's instructions. Briefly, $500 \mu \mathrm{l}$ MEM supplemented with $10 \%$ FBS was added to the lower chamber as a chemoattractant. The cells that migrated and invaded the underside of the membrane were fixed in $4 \%$ paraformaldehyde and stained with crystal violet, and the cells were counted with the membrane inverted. Simultaneously, tumor cells that passed through the membrane were also counted by the fluorescence method according to the instructions.
Gene intervention via the lentiviral pathway. MG63 OS cells in the logarithmic phase were seeded into 6 -well plates at $1 \times 10^{5}$ cells/well. One day later, lentivirus (Lv-NC, Lv-KLF4, Lv-KLF4+Lv-shRNA-PBB12 and Lv-KLF4+Lv-shRNA-PBB12) was added at a multiplicity of infection (MOI) of 10. The infection efficiency was evaluated by observing the fluorescence of green fluorescent protein (GFP) $72 \mathrm{~h}$ after infection. Total RNA and protein were isolated from the cells and subjected to real-time PCR for the measurement of the levels of PBB12 and hsa-miR-204-5p and western blotting for the measurement of the proteins levels of KLF4, ATF2, cyclin D1, E-cadherin and matrix metalloproteinase-9 (MMP9). In addition, immunofluorescence was used to detect the expression of MMP9 in each group.

Real-time PCR. Total RNA was isolated and reverse-transcribed into cDNA using M-MLV reverse transcriptase. Real-time PCR was performed using the SYBR Premix Ex $\mathrm{Taq}^{\mathrm{TM}}$ kit and TP800 System (Takara), and cDNA (200 ng) was used as the template. The levels of PBB12 and ATF2 were normalized to the level of an endogenous housekeeping gene, $\beta$-actin, using the $2^{-\Delta \Delta C q}$ method (10). For the determination of the hsa-miR-204-5p levels, the level of U6 snRNA was used as the reference. The PCRs were carried out under the following conditions: 40 cycles of denaturation at $95^{\circ} \mathrm{C}$ for $10 \mathrm{sec}$, annealing at $56^{\circ} \mathrm{C}$ for $10 \mathrm{sec}$ and extension at $72^{\circ} \mathrm{C}$ for $10 \mathrm{sec}$. The specific primers used for reverse transcription were 5'-TACCTTGCGAAGTGCTTAAAC-3' for U6 snRNA and 5'-GTCGTATCCAGTGCGTGTCGT GGAGTCGGCAATTGCACTGGATACGAAGGCAT-3' for hsa-miR-204-5p. The PCR primers were as follows: PBB12, 5'-AAGGGCGAATGCAGGCA-3' (forward) and 5'-AAG CAGCCGGCTGGCC-3' (reverse); ATF2, 5'-AATCTCGAC CGCAGTCATT-3' (forward) and 5'-TTAGCTGCTCTTCTC CGACGACC-3' (reverse); $\beta$-actin, 5'-CCTGTACGCCAACAC AGTGC-3' (forward) and 5'-ATACTCCTGCTTGCTGAT CC-3' (reverse); U6 snRNA, 5'-GTGCTCGCTTCGGCAGCA CAT-3' (forward) and 5'-TACCTTGCGAAGTGCTTAAAC-3' (reverse); and miR-204-5p, 5'-GCCGGCGCCCGAGCTCTG GCTC-3' (forward) and 5'-TTCCCTTTGTCATCCTATGCC T-3' (reverse).

Western blotting. Total protein was extracted from cells or tissues using the M-PER mammalian protein extraction reagent or the T-PER tissue protein extraction reagent (78501/78510, Pierce; Thermo Fisher Scientific, Inc.). Equal amounts of total protein $(16 \mu \mathrm{g})$ were loaded onto SDS-PAGE gels (11\%) and transferred onto nitrocellulose membranes. The blots were probed with the primary antibodies against human KLF4 (cat. no. ab215036, dilution 1:200), ATF2 (cat. no. ab32019, dilution 1:500), cyclin D1 (cat. no. ab137867, dilution 1:350) and $\beta$-actin (cat. no. ab179467, dilution $1: 12,00)($ Abcam), followed by probing with the secondary HRP-conjugated anti-rabbit/mouse antibody (cat. no. ab6721, 1:3,000; cat. no. ab205719, 1:4,000; Abcam). After washing, the bands were detected by chemiluminescence and imaged with X-ray film which was used to detect the bands, and the relative optical densities were analyzed using image processing software TotalLab v1.10 (TotalLab, Ltd.). $\beta$-actin was used as an endogenous reference for normalization. 
Immunofluorescence (IF). For immunofluorescence, the cells were fixed with $4 \%$ formaldehyde, permeabilized with $0.4 \%$ Triton X-100, incubated with $10 \%$ donkey serum, and stained with anti-MMP9 (cat. no. ab38898, dilution 1:400) overnight at $4^{\circ} \mathrm{C}$. Hoechst 33342 (cat. no. H21492; Invitrogen; Thermo Fisher Scientific, Inc.) was used for nuclear DNA staining.

Statistical analysis. Data are shown as the mean \pm SD of three independent experiments. All statistical data were analyzed using SPSS GradPack version 20.0 statistical software (IBM Corp.) and GraphPad Prism 7.0 (GraphPad Software, Inc.). Comparisons between groups were analyzed using a two-tailed Student's t-test or one-way ANOVA with a post hoc Tukey test. Differences were considered to be statistically significant at $\mathrm{P}<0.05$.

\section{Results}

Differential expression analysis of KLF4, ATF2, hsa-miR204-5p and PBB12 during the process of OS metastasis. KLF4 protein detection data showed that regardless of whether OS metastasis occurred, KLF4 expression in tumors was significantly lower than that in adjacent tissues $(\mathrm{P}<0.01 \mathrm{vs.}$ autologous adjacent tissues). There was no significant difference in KLF4 protein expression in OS without metastasis and OS with metastasis ( $\mathrm{P}>0.05$; Fig. 1A). ATF2 protein showed an opposite trend of KLF4; that is, the ATF2 expression levels were significantly higher in both types of OS than in adjacent tissues $(\mathrm{P}<0.01$ vs. autologous adjacent tissues). In contrast to that of KLF4, the expression of ATF2 was significantly higher in OS with metastasis than in OS without metastasis $(\mathrm{P}<0.01$ vs. osteosarcoma without metastasis; Fig. 1B). Real-time PCR data indicated that there were no significant differences in the ATF2 mRNA levels in all groups ( $\mathrm{P}>0.05$; Fig. 1C). Real-time PCR results also showed that the hsa-miR-204-5p content in two types of OS and the adjacent tissues was completely consistent with the changes in the KLF4 protein (Fig. 1D). The level of PBB12 was significantly higher in OS with metastasis and adjacent tissues than that in OS without metastasis and adjacent tissues $(\mathrm{P}<0.01$, vs. non-metastatic osteosarcoma and adjacent tissues), but there was no significant difference in the PBB12 level in tumors and autologous adjacent tissues in either type of OS (P>0.05; Fig. 1E). Real-time PCR data showed that there was no difference in the PBB12 content of OS and adjacent tissues regardless of whether metastasis occurred; however, the content of PBB12 was significantly higher in metastatic tumors and adjacent tissues than in nonmetastatic tumors and adjacent tissues, and this result indicated that PBB12 was highly expressed in patients with metastatic OS. According to the available data, it is highly probable that the content of PBB12 does not change significantly with time in the progression of OS, and this hypothesis is completely consistent with the conclusion of this study. In the case of high PBB12 expression, there was no correlation between KLF4 and ATF2, and in the case of low PBB12 expression, the two proteins showed a negative correlation; this correlation is one of the reasons for the existence of the KLF4/hsa-miR-204-5p/ATF2 regulatory pathway. That is, the metastasis probability for OS patients with high expression of PBB12 is significantly increased, and PBB12 has the potential to become a screening marker for high-risk groups with metastatic OS.
Luciferase experiment. The verification of the miR-204-5p seed region in the 3'UTR of ATF2 was performed as follows: 'TargetScan 7.1' (http://www.targetscan.org/) analysis showed that the 3'UTR region of the ATF2 gene contained a 7-bp seed region (5'-AAGGGAA-3') that could be bound by hsa-miR-204-5p. After cotransfection of $293 \mathrm{~T}$ cells for $48 \mathrm{~h}$, a luciferase activity assay showed that the hsa-miR-204-5p-mimic significantly decreased the luciferase activity in cells transfected with the wild-type luciferase reporter vector from $33.51 \pm 3.13$ to $15.08 \pm 1.24$ and that the hsa-miR-204-5p-mimic significantly increased the luciferase activity in cells transfected with the wild-type luciferase reporter vector from $33.51 \pm 3.13$ to $59.32 \pm 8.61$. The differences between all cotransfection groups and the group transfected with pGL3-mt-ATF2 were not significant (Fig. 2A).

PBB12 significantly inhibits the binding of hsa-miR-204-5p via the seed region to the 3'UTR region of the ATF2 gene. We used the software 'TargetScan' to predict the binding sites of PBB12 and hsa-miR-204-5p. The results showed that there were at least three hsa-miR-204-5p seed regions in PBB12, and the positions were distributed over 60 bases. The luciferase activity assay performed $48 \mathrm{~h}$ after the cotransfection of the 293T cells showed that the luciferase activity was significantly lower in the miR-204-5p-mimic- and pGL3-wt-ATF2-cotransfected cells than in the pGL3-wt-ATF2-transfected cells $(\mathrm{P}<0.01$ vs. the pGL3-wt-ATF2-transfected group). There was no significant change in the luciferase activity in the pcDH1-PBB12- or pcDH1 vector- and pGL3-wt-ATF2cotransfected cells $(\mathrm{P}<0.01$, vs. pGL3-wt-ATF2-transfected group) compared with that in the miR-204-5p-mimic- and pGL3-wt-ATF2-cotransfected cells. There was a significant increase in the luciferase activity in the miR-204-5p-mimicand pGL3-wt-ATF2 - and pcDH1-PBB12-cotransfected cells $(\mathrm{P}<0.01$ vs. miR-204-5p-mimic- and pGL3-wt-ATF2cotransfected cells) (Fig. 2B).

Analysis of the regulation of the transcription of hsa-miR-204-5p by KLF4 was performed as follows. To identify the hsa-miR-204-5p promoter, the hsa-miR-204-5p precursor pri-miR-204 sequence in the human genome was first determined. The $2.5-\mathrm{kb}$ promoter sequence upstream of the transcription initiation sites was identified and used to predict the location of the promoter sequence by using Promoter 2.0 prediction software. The prediction results showed that the promoter was a 432-bp DNA sequence. 'JASPAR' predicted that KLF4 bound to the theoretical TFBS in the pri-miR-204 promoter. TFBS was validated using a luciferase reporter gene assay. After $48 \mathrm{~h}$ of cotransfection in 293TN cells, overexpression of KLF4 (pcDH1-KLF4 transfection) significantly activated the luciferase activity in cells transfected with pGL3-wt(TFBS)-pro-miR204 $(\mathrm{P}<0.01$, vs. pGL3-wt(TFBS)-pro-miR204-transfected cells) but did not have a significant effect on the luciferase activity in cells transfected with pGL3-mt(TFBS)-pro-miR204 (P>0.05, vs. pGL3-wt(TFBS)-pro-miR204-transfected cells) (Fig. 2C).

Silencing and overexpression of $P B B 12$ enhance and reverse the inhibition of KLF4 during OS cell proliferation and invasion. MG63 and SAOS-2 OS cells were reseeded $72 \mathrm{~h}$ after infection. The proliferative activity analysis showed that KLF4 gene overexpression (Lv-KLF4) significantly inhibited the proliferative activity of MG63 and SAOS-2 

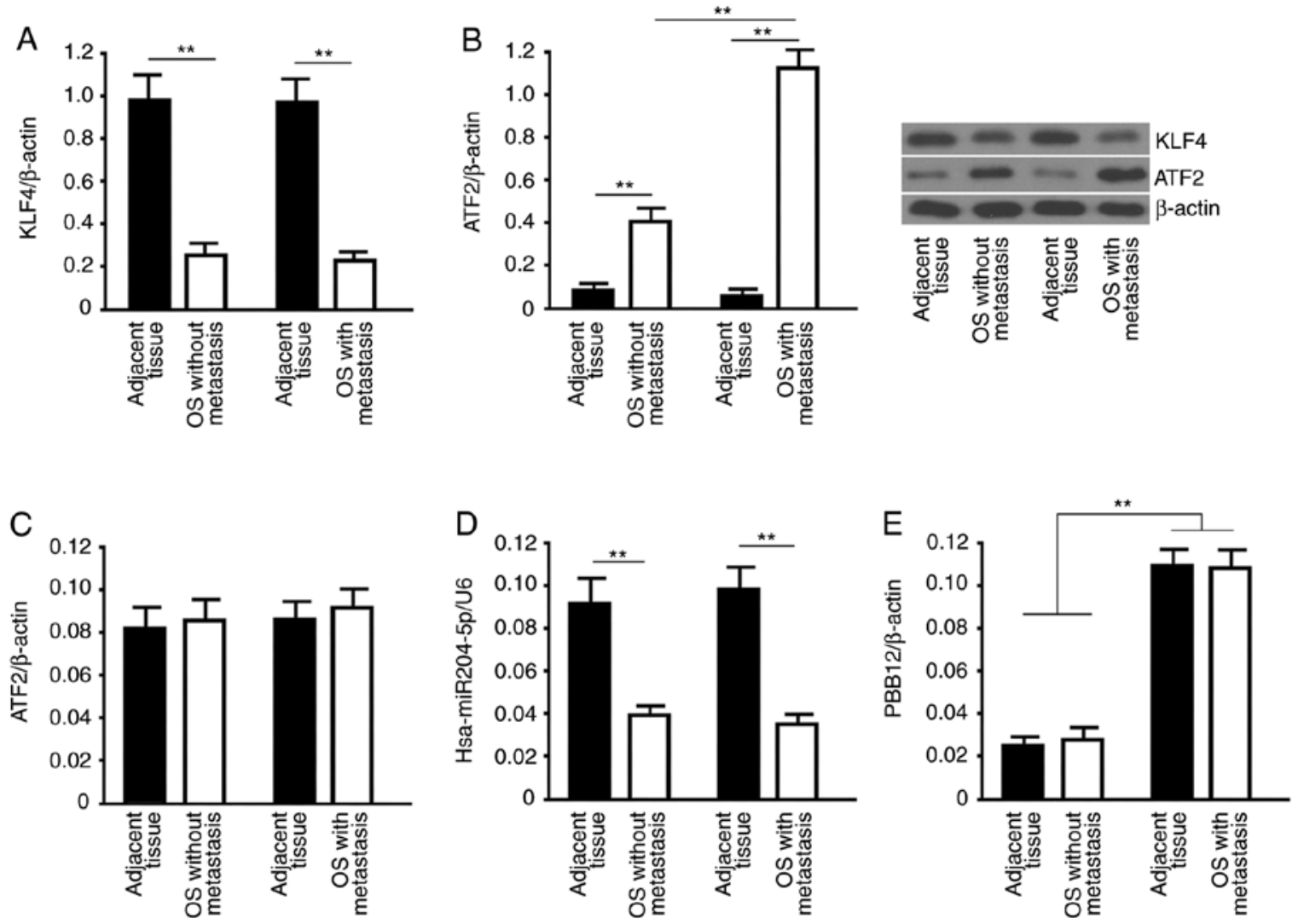

Figure 1. Analyses of KLF4, ATF2, hsa-miR-204-5p and PBB12 in OS and adjacent tissues. Analyses of the levels of (A) KLF4 and (B) ATF2 in OS tissues with and without metastasis and adjacent tissues were carried out by western blot analysis. For western blot analysis, $\beta$-actin was used as the internal control protein. The target band sizes of KLF4, ATF2 and $\beta$-actin proteins were 54, 49 and $37 \mathrm{kDa}$, respectively. Using real-time PCR, the relative values of (C) ATF2 mRNA, (D) hsa-miR-204-5p and (E) PBB12 in OS tissues with and without metastasis and adjacent tissues were analyzed using the $2^{-\Delta \Delta C q}$ method. $\beta$-actin (for the RNA levels of ATF2 mRNA and PBB12) or U6 (for the hsa-miR-204-5p level) were used as the internal controls. All data are expressed as the mean \pm SD The sample size of the group was $n=12 .{ }^{* *} \mathrm{P}<0.01$, t-test. OS, osteosarcoma; KLF4, Kruppel-like factor 4; ATF2, activating transcription factor 2.

cells in the logarithmic phase $(\mathrm{P}<0.01$, vs. the control group or the NC group, $72 \mathrm{~h}$ ). Proliferation was further inhibited in the KLF4 overexpression and PBB12 silencing (Lv-shRNA-PBB12+LV-KLF4) groups ( $<<0.05$, vs. the KLF4 overexpression group, $72 \mathrm{~h}$ ), while the proliferation activity in the KLF4 overexpression and PBB12 overexpression (Lv-PBB12+Lv-KLF4) groups was significantly increased $(\mathrm{P}<0.01$, vs. the KLF4 overexpression group, 72 h) (Fig. 3A). The tumor cell invasion assay showed that overexpression of the KLF4 gene (Lv-KLF4) significantly inhibited the invasion of MG63 and SAOS- 2 cells $(\mathrm{P}<0.01$, vs. the control group or the NC group); PBB12 silencing (Lv-shRNA-PBB12) significantly enhanced the inhibitory effect of KLF4 on tumor cell invasion ( $\mathrm{P}<0.05$, vs the KLF4 overexpression group), while PBB12 overexpression (Lv-PBB12) significantly abrogated the inhibitory effect of the KLF4 gene on invasion in the overexpression group $(\mathrm{P}<0.01$, vs. the KLF4 overexpression group; $\mathrm{P}>0.05$, vs. the control group or the NC group) (Fig. 3B).

PBB12 affects downstream functional proteins in the KLF4/hsa-miR-204-5p/ATF2 pathway during OS metastasis. The KLF4 protein detection assay showed that infection with Lv-KLF4 upregulated KLF4 protein expression in the MG63 cells ( $\mathrm{P}<0.01$ vs. the control group or the NC group), and there were no significant changes in KLF4 expression between the Lv-KLF4-, Lv-PBB12+Lv-KLF4- and
Lv-shRNA-PBB12+Lv-KLF4-infected groups $(\mathrm{P}>0.05)$. The ATF2 protein detection assay showed that ATF 2 protein expression was significantly decreased in the Lv-KLF4-transfected MG63 cells ( $\mathrm{P}<0.05$, vs. the control group or the NC group), and ATF2 was also significantly decreased in the Lv-PBB12or Lv-shRNA-PBB12+Lv-KLF4-transfected MG63 cells ( $\mathrm{P}<0.01$, vs. the control group or the $\mathrm{NC}$ group). The expression of cyclin D1 and MMP9 was consistent with that of ATF2 in all groups. The expression of the E-cadherin protein in each group was the complete opposite of that of ATF2 and cyclin D1 in all groups (Fig. 4A). Immunofluorescence staining of MMP9 showed that the overexpression of KLF4 significantly inhibited the expression of the MMP9 protein in MG63 cells. The silencing or overexpression of PBB12 significantly enhanced or reduced the inhibition of KLF4 on the upregulation of the MMP9 protein (Fig. 4B).

\section{Discussion}

Long non-coding RNA (lncRNA) generally refers to endogenous RNA sequences with a length greater than $200 \mathrm{bp}$. In recent years, many lncRNAs have been discovered and are attracting research attention. These lncRNAs have no protein-coding function; however, they can take part in many types of life-sustaining activities by regulating epigenetic modifications. Compared with genes that encode proteins, 


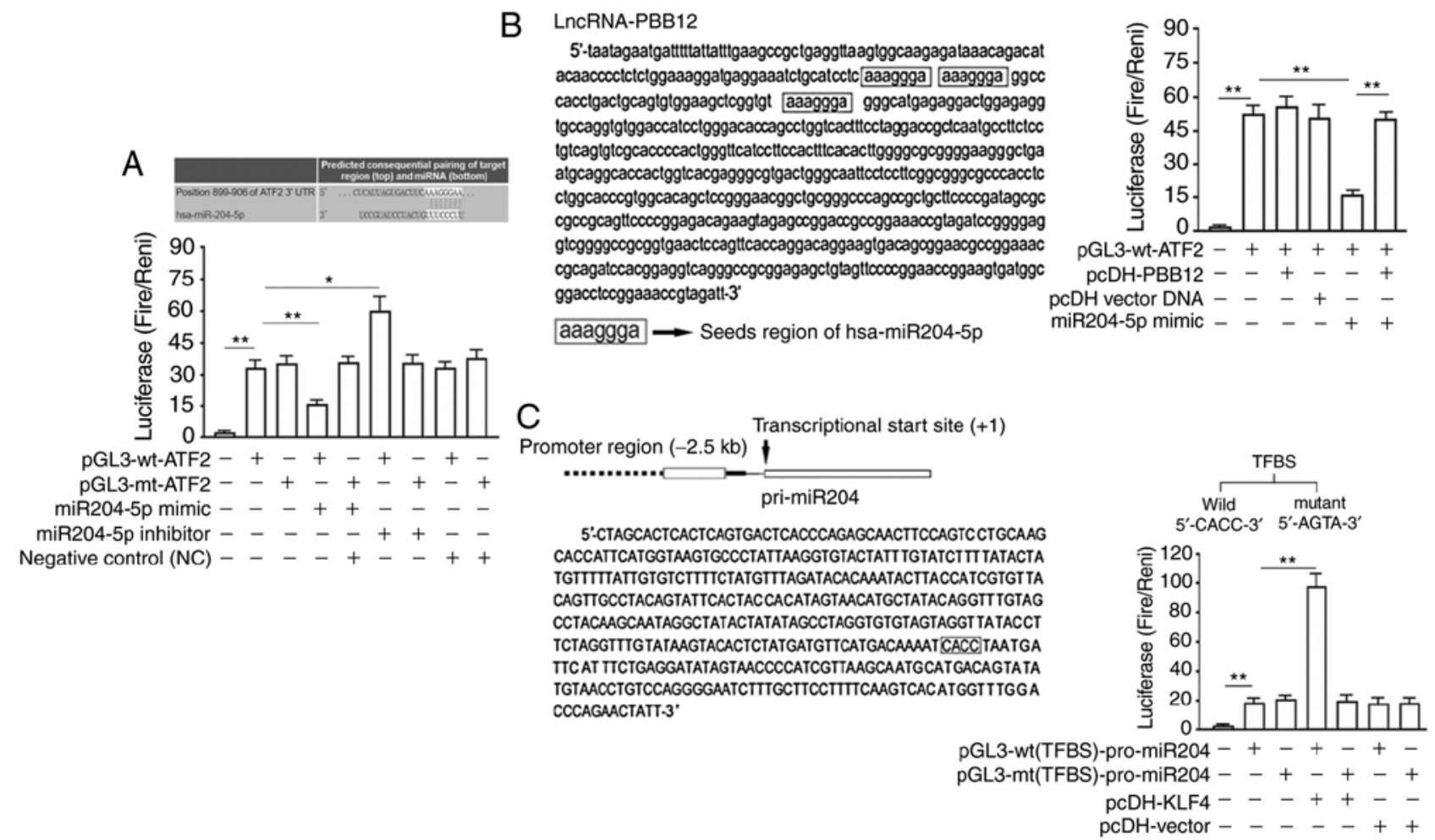

Figure 2. Luciferase assay. (A) Prediction of the binding site of hsa-miR-204-5p in the 3'UTR region of the ATF2 gene and the detection of luciferase activity. Luciferase activity was detected after $48 \mathrm{~h}$ of cotransfection in 293TN cells. (B) Bioinformatic analysis and luciferase assays showed that PBB12 was competitively compatible with miR-204-5p. (C) Analysis of the promoter sequences of pri-miR-204 as well as bioinformatic analysis and luciferase assays identifying the TFBS of KLF4 in the promoter of pri-miR-204. All data are expressed as the mean \pm SD, and the tests were carried out with three biological triplicates. ${ }^{*} \mathrm{P}<0.05$ and ${ }^{* *} \mathrm{P}<0.01$, t-test. KLF4, Kruppel-like factor 4; ATF2, activating transcription factor 2; TFBS, transcription factor binding site.

lncRNAs are large in quantity. lncRNAs can be considered important regulatory molecules in the human genome and have many biological functions. They are involved in chromosome silencing, chromatin modification, transcription regulation and other biological processes $(11,12)$. IncRNAs interact with microRNAs (miRNAs), and this interaction has an important influence on tumor progression (13). miRNAs can target and regulate lncRNAs. miR-21 was found to not only target and inhibit its target protein gene but also IncRNA-GAS5. Furthermore, lncRNAs can also affect the tumor regulation function of miRNAs in several ways (14-16).

The role of miRNAs in molecular regulatory mechanisms involved in osteosarcoma (OS) has attracted much research attention $(17,18)$. Studies have shown that the content of miR-21 in OS is significantly increased. Moreover, the proliferation, invasion and metastasis of MG63 OS cells were found to be promoted by the negative regulation of the expression of the tumor-suppressor gene RECK $(19,20)$. miR-199a-3p is significantly reduced in OS. Overexpression of miR-199a-3p can halt the progression of tumor cells in the cell cycle in the G1 phase, and the growth and migration of tumor cells are inhibited by the negative regulation of the expression of STAT3, the target protein of miR-199a (21). Other studies have shown that through miR-125b and its target protein STAT3, a closed positive feedback pathway can be generated that jointly regulates the proliferation and migration of MG63 and SAOS-2 OS cells and their development into subcutaneous tumors (22). miR-143-3-p, miR-544, miR-382 and miR-369-3p have all been indicated to indirectly transcriptionally regulate their downstream transcription product miR-17-5p through the negative regulation of the expression of the common target gene MYC. Finally, apoptosis of SAOS-2 OS cells is affected (23). Let-7 and miR-564 have been shown to inhibit and promote OS cell invasion, respectively $(24,25)$. All of these studies show that miRNAs can participate in the regulation of OS in many ways.

The interaction between miRNAs and IncRNAs in the cancer field has been studied, and this will lead to important changes in our understanding of the structural networks and regulatory networks of tumor cells (26). This has inestimable scientific and clinical value (27). The lncRNA TUG1 has been proven to bind to miR-9-5p and miR-335-5p as a competitive endogenous RNA to inhibit the negative regulation of the expression of the functional genes POU2F1 and ROCK1. Ultimately, OS cell proliferation and invasion and apoptosis inhibition are promoted (28-30). The IncRNAs PVT1 and H19 have been shown to promote the progression of OS by causing the functional inactivation of members of the miR-19 and miR-200 families $(31,32)$. Studies have shown that the synthesized pyrrolidine polyamide (Myc-6) can be used to partially downregulate MALAT1 levels and inhibit the progression of OS. The mechanism of action may be related to the inhibition of miR-376a function and the upregulation of the expression of TGF- $\alpha$, the target protein of miR-376a (33). These studies have shown that IncRNAs affect miRNA function, which is generally involved in the regulation of tumor progression.

hsa-miR-204-5p is located on human chromosome 9. Previous studies have shown that hsa-miR-204-5p is a 

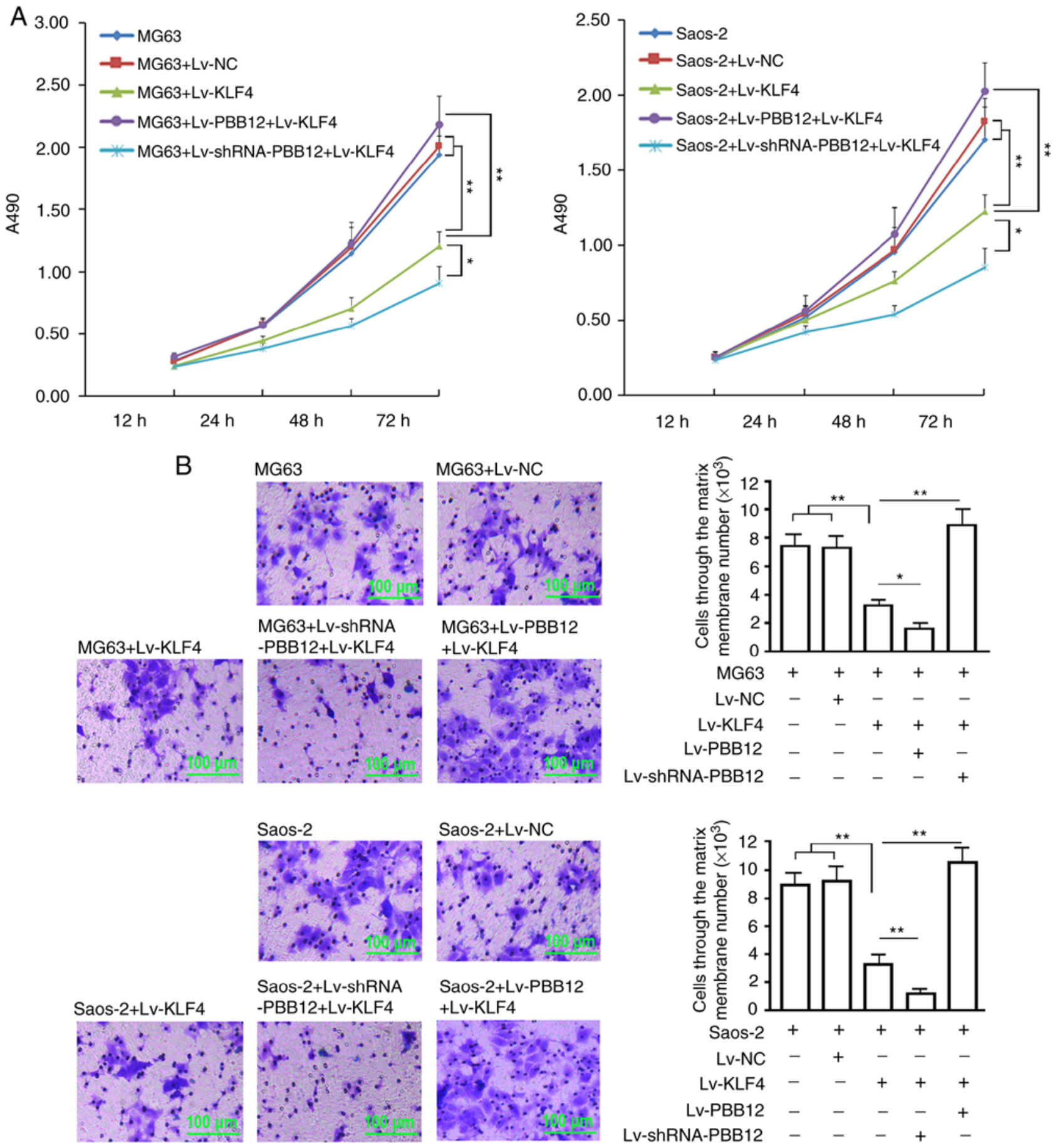

Figure 3. Cell proliferation and invasion assay. (A) MG63 and SAOS-2 cells were infected with the indicated lentiviruses (Lv-NC,Lv-KLF4,Lv-PBB12+Lv-KLF4 or Lv-shRNA-PBB12+Lv-KLF4), seeded into 96-well plates and subjected to a cell viability assay at the indicated times. (B) Invasion data from MG63 and SAOS- 2 cells $72 \mathrm{~h}$ after being infected with the indicated viruses (Lv-NC, Lv-KLF4, Lv-PBB12+Lv-KLF4 or Lv-shRNA-PBB12+Lv-KLF4), as determined by a Transwell assay. The crystal violet-stained cells are the cells that passed through the membrane, and the counts reflect the number of stained cells that passed through the membrane, which were estimated using the absorbance and the standard curve. The x-coordinate represents the cell grouping, and the y-coordinate represents the cell number. ${ }^{* *} \mathrm{P}<0.01,{ }^{*} \mathrm{P}<0.05$. The tests were carried out with three biological triplicates, and the data are expressed as the mean $\pm \mathrm{SD}$. KLF4, Kruppel-like factor 4; ATF2, activating transcription factor 2.

tumor-suppressor gene in many tumor types. Luan and others have shown that hsa-miR-204-5p can target MMP9 and BCL-2. Therefore, it functions as a tumor-suppressor gene in malignant melanoma (34). Research by Sümbül et al revealed that miR-204-5p is increased in colorectal cancer to inhibit the increased activity of LC3B-II in autophagy and Bcl2 against apoptosis post-transcriptionally and acts as a tumor suppressor (35). hsa-miR-204-5 has also been shown to affect the progression of breast cancer through the regulation of the expression of its target protein SIX1 (36). However, there have been no relevant reports on hsa-miR-204-5p and OS progression.

In the present study, low expression of hsa-miR-204-5p in OS tissues was first identified. This directly caused high 

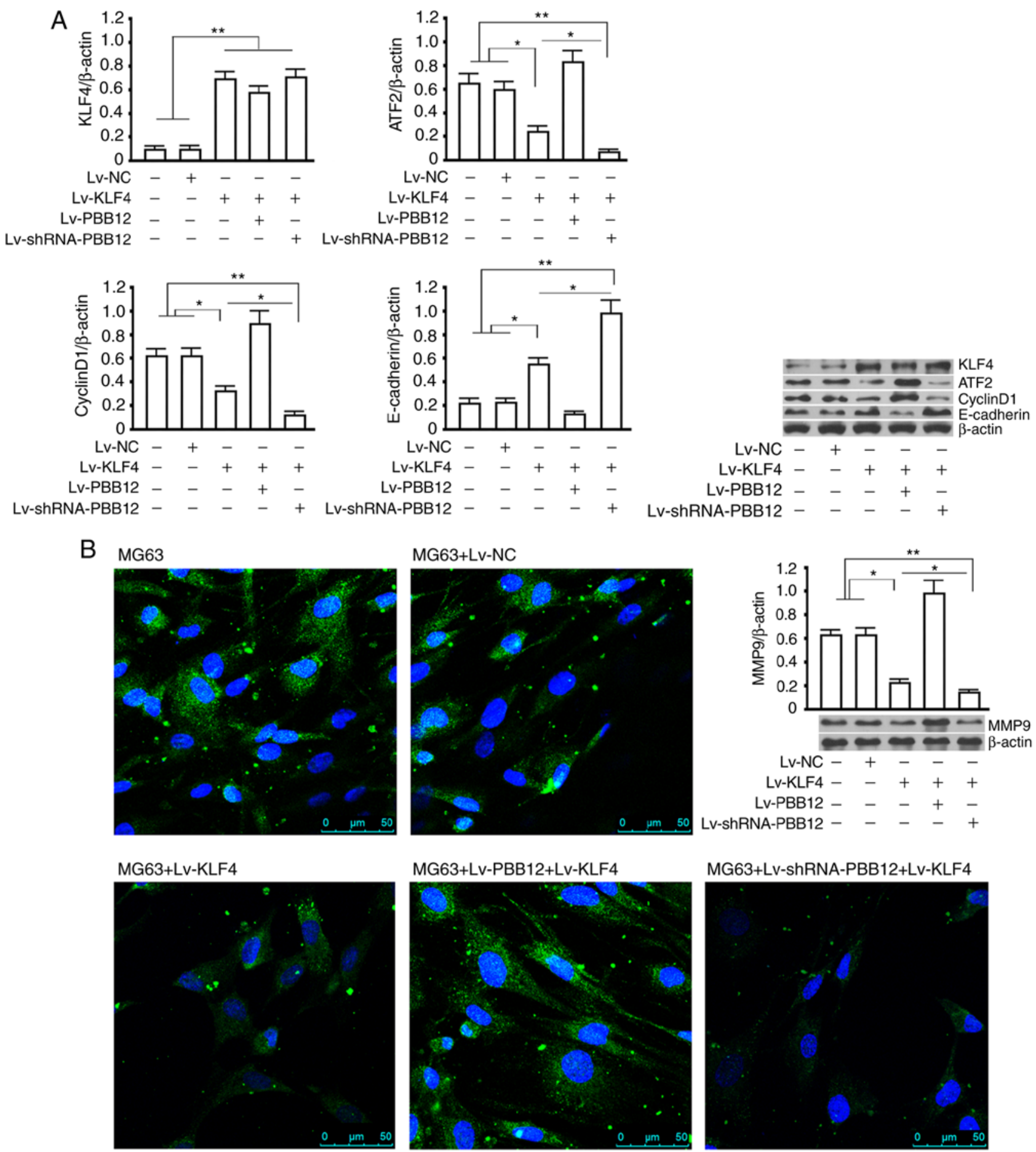

Figure 4. Western blotting and immunofluorescence assays for functional proteins downstream of the KLF4/hsa-miR-204-5p/ATF2 pathway. (A) Western blotting of KLF4 $(54 \mathrm{kDa})$, ATF2 $(69 \mathrm{kDa})$, cyclin D1 $(33 \mathrm{kDa})$, MMP9 $(84 \mathrm{kDa})$ and E-cadherin $(120 \mathrm{kDa})$ in all groups after $72 \mathrm{~h}$ of recombinant virus infection (Lv-NC, Lv-KLF4, Lv-PBB12+Lv-KLF4 or Lv-shRNA-PBB12+Lv-KLF4). $\beta$-actin was used as the internal control. (B) Immunofluorescence staining of the MMP9 protein in MG63 cells from each group after $72 \mathrm{~h}$ of recombinant virus infection (Lv-NC, Lv-KLF4, Lv-PBB12+Lv-KLF4 or Lv-shRNA-PBB12+Lv-KLF4). Blue represents Hoechst staining, and green represents the FITC-labeled MMP9 primary antibody. "P<0.05 and ${ }^{* *} \mathrm{P}<0.01$. The tests were carried out with three biological triplicates, and the data are expressed as the mean \pm SD. KLF4, Kruppel-like factor 4; ATF2, activating transcription factor 2; MMP9, matrix metalloproteinase-9.

expression of the oncogene $A T F 2$. Interestingly, the comparative analysis of the hsa-miR-204-5p content in non-metastatic OS and metastatic OS tissues showed that its expression was low in both groups of tumors, and there was no difference between the groups. However, the expression of its target protein ATF2 in both groups of tumor tissues showed an opposite trend. To explain this phenomenon, transcription factors upstream of hsa-miR-204-5p were screened and predicted. Finally, it was confirmed that the nuclear transcription factor KLF4 can bind to the hsa-miR-204-5p promoter and positively regulate its transcription. Surprisingly, regulation by the upstream regulatory nuclear transcription factor 
KLF4 of hsa-miR-204-5p appeared to be unrelated to OS metastasis. However, the expression of KLF4 in the two groups of tumors and their adjacent tissues was completely consistent with that of hsa-miR-204-5p. These phenomena indicate that the regulatory pathway involved in the expression of KLF4/hsa-miR-204-5p/ATF2 is involved in the progression of OS metastasis. The comparative analysis of the data obtained from the study of metastatic and non-metastatic OS showed that there were no obvious changes in the content of KLF4 and hsa-miR-204-5p in both groups of tumor tissues. However, the expression of the ATF2 protein was significantly altered. Its content in metastatic tumor tissue was significantly greater than that in the non-metastatic tumor tissue. At this point, we believe that there are other factors involved in the abovementioned pathway. The expression of the ATF2 protein was further increased, which contributed to tumor metastasis.

ATF2 belongs to the basic leucine zipper domain bZIP transcription factor family. Under stress conditions, p38/JNK kinase can directly phosphorylate the Thr69 and Thr71 sites in the ATF2 protein; thus, ATF2 transcriptional activity is activated. Phosphorylated ATF2 can form homodimers or heterodimers and bind to specific DNA sequences. The expression of target genes is thereby regulated (37). Studies have shown that ATF2 has dual functions in carcinogenesis and cancer suppression during the tumorigenesis of different types of tumors. In addition to being related to the specific tissue/cell types used in different studies, the intracellular localization of ATF2 may be key to its role in tumorigenesis (38). ATF2 can bind to the cyclin D1 promoter. However, its transcription level is upregulated, and breast cancer progression is promoted (39). Fan and others have shown that ATF2 can promote the proliferation and invasion of tumor cells through the transcriptional regulation of MMP2 (40). Xu et al also confirmed that ATF2 can affect the invasive capability of pancreatic tumor cells through the regulation of the expression of E-cadherin (41). Yet, there have only been a few studies on the relationship between the ATF2 gene and OS. Only a few studies have shown that ATF2 is highly expressed in OS (42). However, there have been no reports on the specific underlying mechanism. In the present study, we believe that the transcriptional regulation mechanism of ATF2 during metastasis of OS is deactivated, which explains why its expression in OS tissues was significantly higher than that in adjacent tissues, and its protein expression was significantly higher in metastatic OS than in non-metastatic OS. Furthermore, the transcription level of the ATF 2 gene showed no obvious change in the two groups of tumor specimens. We believe that the cause of the abnormal expression of ATF2 may be the abnormal function of miRNAs, which function as part of a classical posttranscriptional regulatory mechanism.

In summary, metastasis is the main reason for the poor prognosis of OS patients. OS treatment must be based on inhibition of tumor metastasis. Fortunately, after systematic and in-depth research, lncRNA PBB12 was found to bind to hsa-miR-204-5p in the form of an RNA sponge; thus, its negative regulation of ATF2 protein expression was greatly reduced. We believe that this discovery is of great significance for several reasons. First, PBB12 may serve as a marker for screening high-risk osteosarcoma patients with tumor metastasis. The research data provide strong support for this hypothesis. Compared with that from non-metastatic OS patients, the PBB12 content of tumor tissues from metastatic OS patients was significantly increased. However, regardless of the presence of metastasis, the content of PBB12 in tumor tissues was not different from that in the adjacent tissues. The high expression of PBB12 demonstrates the characteristics resulting from individual differences. Second, for the first time, we propose a possible solution for OS metastasis by targeting KLF4. For patients with low expression or deletion of PBB12 in bone or other tissues, KLF4 overexpression can effectively inhibit tumor progression (proliferation and metastasis) via the KLF4/hsa-miR-204-5p/ATF2 pathway; however, for patients with high expression of $\mathrm{PBB} 12$, the inhibition or knockdown of PBB12 may ensure the normal function of the KLF4/hsa-miR-204-5p/ATF2 pathway and the inhibition of OS metastasis.

PBB12 and the members of the KLF4/hsa-miR-204-5p/ ATF2 pathway and their interactions can jointly regulate the mechanisms involved in OS metastasis. However, according to our research, the mechanism and pathway involved in the participation of PBB12 in the progression of OS has been preliminarily explained. In the present study, PBB12 did not directly bind to KLF4. PBB12 only blocked the KLF4/miR-204-5p/ATF2 pathway by binding to miR-204-5p. If PBB12 is regarded as a genetic characteristic of different populations, it is actually a key factor that is independent of KLF4. Therefore, high expression of PBB12 can only change the anticancer properties of KLF4 or restore the anticancer function of KLF4, but it has no correlation with the expression of KLF4. According to the existing theory, PBB12 has no correlation with the expression of any member protein or gene in the KLF4/miR-204-5p/ATF2 pathway. Only when PBB12 is overexpressed does the KLF4/miR-204-5p/ATF2 pathway fail. When PBB12 is underexpressed or deleted, the KLF4/miR-204-5p/ATF2 pathway remains activated. In addition, the expression connection between PBB12 and miR-204-5p also should be further studied. According to our existing theory, PBB12 binds to miR-204-5p and inhibits the latter binding to the 3 'UTR of the $A T F 2$ gene, ultimately inhibiting the expression of the ATF2 protein. However, it is unlikely that there is a correlation between PBB12 and miR-204-5p. In fact, the test data of clinical samples also showed no correlation between PBB12 and miR-204-5p, and the results support the fact that miRNAs inhibit the translation of target genes into proteins by binding to the 3'UTR region of the target gene. Whether miRNAs have an effect on the content of target genes is not fully accepted in the current mainstream view. In theory, there is no influence (miRNAs are believed to inhibit translation, not degrade transcripts, and the classical theory is that the target genes regulated by miRNAs are posttranscriptionally regulated); however, there are many reports on the decrease in RNA content. Some scholars believe that miRNAs have no effect on the contents of target genes. The decrease in the target gene content results from feedback regulation $(43,44)$.

Although our study initially explained that PBB12 is involved in the progression of OS, future research still need to be carried out. Over a long period of time, the large-scale verification of the differences in the expression of PBB12 in OS patients and the explanation for these expression differences require more in-depth and systematic research. In 
addition, the addition of in vivo research would have made the present study more convincing. The main reasons why we did not formally carry out animal experiments in vivo were as follows: i) The effect of PBB12 on the subcutaneous tumor-bearing volume can be observed through subcutaneous tumor-bearing experiments; however, these experiments are not suitable for observing tumor metastasis; ii) the expression of PBB12 has certain population characteristics, but its status in nude mice remains unclear. In addition, the expression also involves the homology of human and mouse PBB12 sequences. Based on the above, we are preparing and implementing two in vivo studies. We will first establish a mouse PDX model of OS and second, aim to establish PBB12 knock-in mice and then establish a PDX model to avoid the concerns caused by the homology of human and mouse PBB12 (if the homology between mouse PBB12 and human PBB12 is not ideal). We believe such animal experiments will undoubtedly be more meaningful. The significance of the present study is the revelation that some cancer suppression regulatory pathways may lose their tumor-suppressive function in specific patient populations due to particular lncRNAs. Our study confirmed that PBB12 has the potential to serve as a marker for screening high-risk OS patients for tumor metastasis. In addition, for patients with high expression of PBB12, PBB12 can be inhibited and/or knocked down, which may ensure normal function of the KLF4/hsa-miR-204-5p/ATF2 cancer suppression pathway. Furthermore, the anticancer function of the KLF4 gene can be utilized. This evidence provides strong theoretical support for the improvement of gene therapy for OS by targeting KLF4.

\section{Acknowledgements}

Not applicable.

\section{Funding}

This research was supported by the National Natural Science Foundation of China (81772267 and 81072070).

\section{Availability of data and materials}

The datasets used and/or analyzed during the current study are available from the corresponding author on reasonable request.

\section{Authors' contributions}

TM performed the experiments and contributed in drafting the manuscript. AL and DX performed the analysis and interpreted the data. TZ conceived and designed the study. All authors read and approved the final version of the manuscript and agree to be accountable for all aspects of the work in ensuring that questions related to the accuracy or integrity of any part of the work are appropriately investigated and resolved.

\section{Ethics approval and consent to participate}

This study was approved by the Ethics Review Committee of Huashan Hospital Affiliated to Fudan University (2016-037), and written informed consent was obtained from all participants and/or their guardians.

\section{Patient consent for publication}

Not applicable.

\section{Competing interests}

The authors declare that they have no competing interests, and all authors confirm its accuracy.

\section{References}

1. Kasinski AL and Slack FJ: Epigenetics and genetics. MicroRNAs en route to the clinic: Progress in validating and targeting microRNAs for cancer therapy. Nat Rev Cancer 11: 849-864, 2011.

2. Liang W, Gao B, Fu P, Xu S, Qian Y and Fu Q: The miRNAs in the pathgenesis of osteosarcoma. Front Biosci (Landmark Ed) 18: 788-794, 2013.

3. Hauptman $\mathrm{N}$ and Glavač D: Long non-coding RNA in cancer. Int J Mol Sci 14: 4655-4669, 2013.

4. Abdelmohsen $\mathrm{K}$ and Gorospe M: Noncoding RNA control of cellular senescence. Wiley Interdiscip Rev RNA 6: 615-629, 2015.

5. Alaei-Mahabadi B and Larsson E: Limited evidence for evolutionarily conserved targeting of long non-coding RNAs by microRNAs. Silence 4: 4, 2013.

6. Zhou DK, Yang XW, Li H, Yang Y, Zhu ZJ and Wu N: Up-regulation of long noncoding RNA CCAL predicts poor patient prognosis and promotes tumor metastasis in osteosarcoma. Int J Biol Markers 32: e108-e112, 2017.

7. Li W, Xie P and Ruan WH: Overexpression of lncRNA UCA1 promotes osteosarcoma progression and correlates with poor prognosis. J Bone Oncol 5: 80-85, 2016.

8. Fan H, Liu G, Zhao C, Li X and Yang X: Transcription factor Oct4 promotes osteosarcoma by regulating lncRNA AK055347. Oncol Lett 13: 396-402, 2017.

9. Fornes O, Castro-Mondragon JA, Khan A, van der Lee R, Zhang X, Richmond PA, Modi BP, Correard S, Gheorghe M, Baranašić D, et al: JASPAR 2020: Update of the open-access database of transcription factor binding profiles. Nucleic Acids Res: Nov 8, 2019 (Epub ahead of print).

10. Livak KJ and Schmittgen TD: Analysis of relative gene expression data using real-time quantitative PCR and the 2(-Delta Delta C(T)) method. Methods 25: 402-408, 2001.

11. Ponting CP, Oliver PL and Reik W: Evolution and functions of long noncoding RNAs. Cell 136: 629-641, 2009.

12. Bergmann JH and Spector DL: Long non-coding RNAs: Modulators of nuclear structure and function. Curr Opin Cell Biol 26: 10-18, 2014

13. Deng G and Sui G: Noncoding RNA in oncogenesis: A new era of identifying key players. Int J Mol Sci 14: 18319-18349, 2013.

14. Pickard MR and Williams GT: Molecular and cellular mechanisms of action of tumour suppressor GAS5 lncRNA. Genes (Basel) 6: 484-499, 2015.

15. Pang M, Xing C, Adams N, Rodriguez-Uribe L, Hughs SE, Hanson SF and Zhang J: Comparative expression of miRNA genes and miRNA-based AFLP marker analysis in cultivated tetraploid cottons. J Plant Physiol 168: 824-830, 2011.

16. Brodersen P, Sakvarelidze-Achard L, Schaller H, Khafif M, Schott G, Bendahmane A and Voinnet O: Isoprenoid biosynthesis is required for miRNA function and affects membrane association of ARGONAUTE 1 in Arabidopsis. Proc Natl Acad Sci USA 109: 1778-1783, 2012.

17. Sampson VB, Yoo S, Kumar A, Vetter NS and Kolb EA: MicroRNAs and potential targets in osteosarcoma: Review. Front Pediatr 3: 69, 2015.

18. Cheng CJ, Bahal R, Babar IA, Pincus Z, Barrera F, Liu C, Svoronos A, Braddock DT, Glazer PM, Engelman DM, et al: MicroRNA silencing for cancer therapy targeted to the tumour microenvironment. Nature 518: 107-110, 2015.

19. Ziyan W, Shuhua Y, Xiufang W and Xiaoyun L: MicroRNA-21 is involved in osteosarcoma cell invasion and migration. Med Oncol 28: 1469-1474, 2011. 
20. Ren X, Shen Y, Zheng S, Liu J and Jiang X: miR-21 predicts poor prognosis in patients with osteosarcoma. Br J Biomed Sci 73: $158-162,2016$.

21. Tian R, Xie X, Han J, Luo C, Yong B, Peng H, Shen J and Peng T: miR-199a-3p negatively regulates the progression of osteosarcoma through targeting AXL. Am J Cancer Res 4: 738-750, 2014

22. Wang F, Yu D, Liu Z, Wang R, Xu Y, Cui H and Zhao T: MiR-125b Functions as a tumor suppressor and enhances chemosensitivity to cisplatin in osteosarcoma. Technol Cancer Res Treat 15 . NP105-NP112, 2016.

23. Thayanithy V, Sarver AL, Kartha RV, Li L, Angstadt AY, Breen M, Steer CJ, Modiano JF and Subramanian S: Perturbation of $14 \mathrm{q} 32 \mathrm{miRNAs-cMYC}$ gene network in osteosarcoma. Bone 50: 171-181, 2012

24. Yu JJ, Pi WS, Cao Y, Peng AF, Cao ZY, Liu JM, Huang SH, Liu ZL and Zhang W: Let-7a inhibits osteosarcoma cell growth and lung metastasis by targeting Aurora-B. Cancer Manag Res 10: 6305-6315, 2018

25. Wang RJ, Shi KR, Zhang J, Zhang J, Gao RR and Zhu SC: Effects of miR-93 on proliferation and apoptosis of osteosarcoma cells. Zhonghua Bing Li Xue Za Zhi 45: 866-870, 2016 (In Chinese).

26. Aftab MN, Dinger ME and Perera RJ: The role of microRNAs and long non-coding RNAs in the pathology, diagnosis, and management of melanoma. Arch Biochem Biophys 563: 60-70, 2014.

27. Xie CH, Cao YM, Huang Y, Shi QW, Guo JH, Fan ZW, Li JG, Chen BW and Wu BY: Long non-coding RNA TUG1 contributes to tumorigenesis of human osteosarcoma by sponging miR-9-5p and regulating POU2F1 expression. Tumour Biol 37: 15031-15041, 2016.

28. Zhang Q, Geng PL, Yin P, Wang XL, Jia JP and Yao J: Down-regulation of long non-coding RNA TUG1 inhibits osteosarcoma cell proliferation and promotes apoptosis. Asian Pac J Cancer Prev 14: 2311-2315, 2013.

29. Wang $Y$, Yang T, Zhang Z, Lu M, Zhao W, Zeng X and Zhang W: Long non-coding RNA TUG1 promotes migration and invasion by acting as a ceRNA of miR-335-5p in osteosarcoma cells. Cancer Sci 108: 859-867, 2017.

30. Zhou Q, Chen F, Zhao J, Li B, Liang Y, Pan W, Zhang S, Wang X and Zheng D: Long non-coding RNA PVT1 promotes osteosarcoma development by acting as a molecular sponge to regulate miR-195. Oncotarget 7: 82620-82633, 2016.

31. Chan LH, Wang W, Yeung W, Deng Y, Yuan P and Mak KK: Hedgehog signaling induces osteosarcoma development through Yap1 and H19 overexpression. Oncogene 33: 4857-4866, 2014

32. Li M, Chen $\mathrm{H}$, Zhao Y, Gao S and Cheng C: H19 functions as a ceRNA in promoting metastasis through decreasing miR-200s activity in osteosarcoma. DNA Cell Biol 35: 235-240, 2016.
33. Taniguchi M, Fujiwara K, Nakai Y, Ozaki T, Koshikawa N, Toshio K, Kataba M, Oguni A, Matsuda H, Yoshida Y, et al: Inhibition of malignant phenotypes of human osteosarcoma cells by a gene silencer, a pyrrole-imidazole polyamide, which targets an E-box motif. FEBS Open Bio 4: 328-334, 2014.

34. Luan W, Qian Y, Ni X, Bu X, Xia Y, Wang J, Ruan H, Ma S and $\mathrm{Xu}$ B: miR-204-5p acts as a tumor suppressor by targeting matrix metalloproteinases-9 and B-cell lymphoma-2 in malignant melanoma. Onco Targets Ther 10: 1237-1246, 2017.

35. Sümbül AT, Göğebakan B, Ergün S, Yengil E, Batmacı CY, Tonyalı Ö and Yaldız M: miR-204-5p expression in colorectal cancer: An autophagy-associated gene. Tumour Biol 35: 12713-12719, 2014.

36. Zeng J,Wei M,ShiR,CaiC,Liu X,LiT and Ma W:MiR-204-5p/Six1 feedback loop promotes epithelial-mesenchymal transition in breast cancer. Tumour Biol 37: 2729-2735, 2016.

37. Vlahopoulos SA, Logotheti S, Mikas D, Giarika A, Gorgoulis V and Zoumpourlis V: The role of ATF-2 in oncogenesis. Bioessays 30: 314-327, 2008.

38. Hsu CC and Hu CD: Critical role of N-terminal end-localized nuclear export signal in regulation of activating transcription factor 2 (ATF2) subcellular localization and transcriptional activity. J Biol Chem 287: 8621-8632, 2012.

39. Lewis JS, Vijayanathan V, Thomas TJ, Pestell RG, Albanese C, Gallo MA and Thomas T: Activation of cyclin D1 by estradiol and spermine in MCF-7 breast cancer cells: A mechanism involving the p38 MAP kinase and phosphorylation of ATF-2. Oncol Res 15: 113-128, 2005

40. Fan Z, Duan X, Cai H, Wang L, Li M, Qu J, Li W, Wang Y and Wang J: Curcumin inhibits the invasion of lung cancer cells by modulating the PKC $\alpha /$ Nox-2/ROS/ATF-2/MMP-9 signaling pathway. Oncol Rep 34: 691-698, 2015.

41. Xu Y, Liu Z and Guo K: The effect of JDP2 and ATF2 on the epithelial-mesenchymal transition of human pancreatic cancer cell lines. Pathol Oncol Res 18: 571-577, 2012.

42. Tang J, Liao Y, He S, Shi J, Peng L, Xu X, Xie F, Diao N, Huang J, $\mathrm{Xie} \mathrm{Q}$, et al: Autocrine parathyroid hormone-like hormone promotes intrahepatic cholangiocarcinoma cell proliferation via increased ERK/JNK-ATF2-cyclinD1 signaling. J Transl Med 15: 238, 2017.

43. Pu M, Li C, Qi X, Chen J, Wang Y, Gao L, Miao L and Ren J: MiR-1254 suppresses HO-1 expression through seed region-dependent silencing and non-seed interaction with TFAP2A transcript to attenuate NSCLC growth. PLoS Genet 13: e1006896, 2017.

44. Zhao Y, Qi X, Chen J, Wei W, Yu C, Yan H, Pu M, Li Y, Miao L, Li C and Ren J: The miR-491-3p/Sp3/ABCB1 axis attenuates multidrug resistance of hepatocellular carcinoma. Cancer Lett 408: 102-111, 2017. 